\title{
Mental Ability in Childhood and Cognitive Aging
}

\author{
Alan J. Gow ${ }^{\mathrm{a}}$ Wendy Johnson ${ }^{\mathrm{a}, \mathrm{c}}$ Alison Pattie $^{\mathrm{a}}$ Martha C. Whiteman ${ }^{\mathrm{a}}$ \\ John Starrb lan J. Deary ${ }^{a}$ \\ Departments of a Psychology and ${ }^{b}$ Geriatric Medicine, University of Edinburgh, Edinburgh, UK; ${ }^{c}$ Department of \\ Psychology, University of Minnesota, Twin Cities, Minn., USA
}

\section{Key Words}

Childhood mental ability $\cdot$ Cognitive aging $\cdot$ Cognitive function

\begin{abstract}
Background: Identifying the determinants of cognitive aging is a research priority; however, few studies are able to examine the influence of pre-morbid cognitive ability on later changes in cognitive function. Objective: To examine the association between childhood cognitive ability and cognitive change from age 79 to 83 in the presence of other demographic and lifestyle indicators. Methods: The participants took a test of mental ability when aged 11 as part of the Scottish Mental Survey 1932. Cognitive ability based on Raven's Matrices, Verbal Fluency, and Logical Memory was assessed at ages 79 and 83 . We used both linear regression and latent variable growth curve modeling to compare methods and results. Results: Using linear regression, childhood mental ability was a significant predictor of cognitive change from 79 to 83 , accounting for about $1.4 \%$ of the variance. Sex, education, social class, smoking status and alcohol intake were non-significant. In contrast, using latent variable growth curve modeling, there was no association between childhood mental ability and cognitive change. Sex (male), years of education, drinking status (positive), and childhood IQ were associated with better cognitive ability at
\end{abstract}

age 79. The difference in results was due to the inability of linear regression to account completely for test-specific variance. Conclusion: Within a group of non-demented older people, greater childhood mental ability was associated with level of cognitive ability at age 79 , but not with change in cognitive ability to age 83 . To obtain accurate results regarding covariates of change, it is important to use methodology that can appropriately allocate all measured sources of variance.

Copyright $\odot 2008$ S. Karger AG, Basel

\section{Introduction}

In identifying determinants of cognitive aging, there is disagreement about whether being initially more able is advantageous [1]. Pre-morbid cognitive ability is a predictor of dementia, whether measured in childhood [2] or early adulthood [3], but few studies have examined the effect of initial ability on normal cognitive aging [4]. The greatest predictor of cognitive ability level in elderly individuals is their prior ability. In one study, childhood ability explained about $50 \%$ of the variance in the same test 68 years later [5]. This does not, however, resolve the issue of whether early ability level is associated with change in later life. Studies rarely possess data to address this, often lacking a valid measure of prior ability.

\section{KARGER \\ Fax +41613061234 E-Mail karger@karger.ch} www.karger.com (c) 2008 S. Karger AG, Basel 0304-324X/08/0543-0177\$24.50/0

Accessible online at: www.karger.com/ger
Ian J. Deary

Psychology, University of Edinburgh

7 George Square

Edinburgh EH8 9JZ (UK)

Tel. +44 131650 3452, Fax +44 131651 1771, E-Mail Ian.Deary@ed.ac.uk 
Some studies have estimated cognitive decline using tasks expected to show little change with age, such as the National Adult Reading Test [6], to measure pre-morbid ability. These tasks are then compared to tasks which display age-associated decrements. In so doing, researchers have stated there is 'no evidence that a greater youthful ability slows the rate at which intelligence test scores decline in later life' [7, p. 70]. However, in the British 1946 birth cohort, lower childhood ability (age 15) predicted greater decline in memory and visual search speed from age 43 to 53, after controlling education and social class [4]. That study assessed cognitive change in middle age, so it is necessary to examine whether any protective effect of higher early ability might be detectable in elderly individuals. In another study, mental ability at age 11 was shown to account for $2 \%$ of the change in Raven's Matrices performance over 2-3 years among 64- and 77-yearolds [8]. The current study investigates cognitive change from 3 tests over 4 years between 79 and 83 . A valid measure of cognitive ability is available from age 11 . We compare results from two methodologies, the frequently-used linear regression, and the potentially more informative latent variable growth curve model that made use of participants with missing data.

\section{Method}

\section{Participants}

The recruitment of the Lothian Birth Cohort 1921 (LBC1921) has been described in detail previously [5]. To summarize, these individuals were given a test of mental ability when aged 11 years as part of the Scottish Mental Survey 1932 (SMS1932). In that survey, almost all Scottish children born in 1921 were tested at school on the same day $(\mathrm{n}=87,498)$ [9]. Surviving participants of the SMS1932 (individuals born in 1921 and at school in Scotland in 1932) from Edinburgh and the surrounding areas were traced either through the Community Health Index or as volunteers replying to media calls. This follow-up began in 1999 and 550 individuals ( 234 men and 316 women) were tested individually by 2 trained researchers.

A second wave of cognitive testing began in 2003 and is reported in detail here for the first time. All retained LBC1921 participants (that is, excluding those individuals who had withdrawn or were known to have died since the previous wave) were sent a letter inviting them to participate in this next stage of the followup, accompanied by a more detailed information leaflet. Mailings were sent to 454 participants, 20 individuals at a time. About 1 week after mailing, the participants were telephoned to determine whether they wished to participate and to make appointments. Transport to and from the session was offered to participants. In total, 119 individuals (26.2\% of those mailed) did not take part in this wave of testing: 10 had died, 16 withdrew from the study after being contacted, 80 were unable or did not wish to participate, and 13 were no longer contactable. Of the rest, 335 participants
(73.8\% of those mailed) agreed to attend the cognitive testing session, and 321 were tested $(70.7 \%$ of those mailed; $58.4 \%$ of the original LBC1921); 45.2\% (145) were male and 54.8\% (176) were female. Testing was conducted by 2 trained researchers in the $\mathrm{Vi}$ sual Psychophysics Laboratory at the Princess Alexandra Eye Pavilion, Royal Infirmary of Edinburgh. Each participant was tested individually in a session lasting between 2.5 and $3.5 \mathrm{~h}$, including breaks.

The mean age of the LBC1921 when tested as children in the Scottish Mental Survey 1932 was 10.9 years $(S D=0.3)$. In late adulthood, the follow-ups occurred at mean ages of $79.1(\mathrm{SD}=0.6)$ and 83.4 years old $(\mathrm{SD}=0.5)$. For simplicity, these are referred to as ages 11,79 and 83 throughout. The mean follow-up time in old age was 4.3 years $(\mathrm{SD}=0.4)$, ranging from 3.1 to 5.8 years.

\section{Measures}

The participants sat the Moray House Test (MHT) at ages 11 and 79. The MHT consists of 71 numbered items, 75 items in total, with a maximum score of 76 . Participants were allowed $45 \mathrm{~min}$ working time [5, 9]. MHT scores at 11 and 79 correlated 0.66 [5], validating the $\mathrm{MHT}$ at age 11 as a measure of prior ability relevant to adulthood. As the stability and validity of this measure across time has been reported previously, and the current paper focuses on the association between childhood ability and later life cognitive change, the age-79 MHT score will not be discussed further. The raw age-11 MHT scores were corrected for age in days at the time of testing and then converted to the IQ score scale (thus by definition, the sample's IQ at 11 had a mean of 100, and an SD of 15).

At ages 79 and 83, participants also completed Raven's Progressive Matrices, Verbal Fluency, and Logical Memory tests. Raven's Matrices consists of 60 items requiring non-verbal, inductive reasoning [10]. Participants were required to choose the correct response (from a choice of 6 or 8 ) to complete a pattern. The LBC1921 were time-limited to $20 \mathrm{~min}$, and performance was the number of correct responses. Verbal Fluency is a measure of executive function [11]. Participants were required to name as many words as possible beginning with a target letter. After a brief practice with the letter S, 1 min was allowed for each of 3 letters (C, F and $\mathrm{L}$ ). The 3 scores were summed to give a Verbal Fluency score. The instructions highlighted that proper nouns and numbers were not acceptable, and that essentially similar words with different endings (for example, see and seeing) would only be counted once [5]. Logical Memory is a subtest of the Wechsler Memory Scale-Revised (WMS-R) [12] assessing verbal declarative memory. Participants were instructed to listen carefully to 2 short stories and, after each, their immediate free recall from memory was assessed. The maximum score was 25 for each story. After a period of time (about $25 \mathrm{~min}$ ), their delayed recall of the stories was assessed. Performance on the immediate and delayed recall of the 2 stories was the number of items correctly recalled. All four scores correlated above 0.57 and so were summed to give a Logical Memory score (maximum score $=100$ ).

To allow a comparison of the sample characteristics for those who did and did not complete the age- 83 wave of testing, the following fitness indicators were recorded at age 79: 6-meter walk time (the time taken in seconds to walk $6 \mathrm{~m}$ at a normal pace); grip strength (measured in kilograms with a Jamar Hydraulic Hand Dynamometer, the best of 3 trials with the dominant hand was used), and $\mathrm{FEV}_{1}$ (the forced expiratory volume from the lungs in 
$1 \mathrm{~s}$ measured with a microspirometer, the best of 3 trials was taken). Participants also completed the Mini-Mental State Examination (MMSE), often used as a brief screening test for dementia [13]. The following demographic and lifestyle information was obtained from each participant: the number of years in full-time, formal education; occupational social class [the highest occupational position achieved, coded according to the Classification of Occupations [14], ranging from 1 (highest) to 5 (lowest); for married women, the social class of their husband was used]; age-79 smoking status (current smoker versus non-smoker, with current smoker as the reference class), and age-79 alcohol intake (participants were asked if they drank alcohol, and if they did, they were further asked to report the type of drink and the quantities of each type consumed in an average week). This information was converted to alcohol units using standard unit values. Weekly alcohol intake as a continuous variable was very skewed in this elderly sample; $52 \%$ reported drinking up to and including 1 unit of alcohol per week. The alcohol intake variable was dichotomised as 1 unit or less per week versus more than 1 , with 1 unit or less as the reference class.

\section{Statistical Analyses}

Age-adjusted scores were created for each of the cognitive ability tests by saving the standardized residual from a linear regression with test performance as the dependent variable and age in days at the time of testing as the independent variable.

\section{Composite Cognitive Ability Score}

To implement the linear regression analyses of cognitive change, a principal components analysis (PCA) was run on the age-adjusted test scores from Raven's Matrices, Verbal Fluency and Logical Memory to determine whether a single ability component appropriately described the data at each wave. In this analysis, Raven's scores for all participants at ages 79 and 83 were combined into a single column in the database; proceeding down each column, the age- 83 scores followed after the age- 79 scores. This process was also carried out for Verbal Fluency and Logical Memory. The PCA was conducted in this way so that a comparison of the means across time would be meaningful. If PCA were conducted separately for ages 79 and 83 , comparison of the mean standardized scores would be uninformative, because means at both ages would be zero. Although this method violates the underlying assumption that the observations are independent it does not bias the resulting principle component loadings; it only overstates the amount of variance accounted for by the component structure [15]. The overall KMO measure of sampling adequacy was 0.60 , with individual test values ranging from 0.57 ( Raven's Matrices) to 0.65 (Verbal Fluency). The scree slope and eigenvalues greater than 1 criteria both suggested that 1 component effectively described the data, accounting for $53.9 \%$ of the variance. The loadings of the cognitive tests on this first unrotated component ranged from 0.66 to 0.80 . The standardized score from the PCA was saved as a composite cognitive ability score. This new variable was then split according to whether it was based on tests completed at ages 79 or 83 .

Linear Regression Analysis of Later Life Cognitive Change

The age- 83 composite cognitive ability score was entered as the dependent variable in a linear regression; the age-79 composite cognitive ability score was entered as the independent variable.
The standardized residual produced was used as a measure of later life cognitive change. The first analyses used it as the dependent variable in linear regression in order to find the contributors to individual differences in cognitive change from age 79 to 83 .

\section{Latent Growth Curve Modeling of Later Life Cognitive \\ Change}

We also implemented a latent variable growth curve model, parameterizing the two sets of cognitive function data as linearly related, thus generating latent intercept and slope terms. Because the component scores used to form our composite cognitive ability variables were based on linear combinations of the observed test scores, they could not completely represent the intended underlying latent, or unobserved, variables consisting of the variance common to the observed variables. Our latent variable growth curve model simultaneously incorporated both the latent variable assessment and the analysis of change, thus eliminating the potential for variance specific to individual tests to bias our results. In addition, it was possible to measure the variances specific to each of the three tests directly, and to measure separately the effects of our demographic and lifestyle variables and age-11 IQ on the level (intercept) of cognitive ability at age 79 and change (slope) in cognitive ability from age 79 to age 83 . We could also measure the correlation between intercept and slope, and the correlations between the latent cognition variables and the demographic and lifestyle variables. Probably most importantly, we could include the participants who were present at age 79 but not at age 83 under the reasonable assumption that they were missing at random, using full information maximum likelihood.

In fitting this model, we could also test an important but often overlooked assumption that underlies the use of the linear regression model. The assumption is that the tests administered measure the same constructs at each age, and it cannot be presumed always to hold. We could test this assumption by allowing the factor loadings, factor intercepts, and factor variances at each age to vary freely in a baseline model and then constraining them to be equal across age in a restricted model. If the restricted model did not fit significantly less well than the baseline model, then it was reasonable to conclude that the tests measured the same constructs in the same way at each age and thus were factorially invariant. This proved to be the case $\left(\Delta \chi^{2}=3.94,8\right.$ d.f., $\left.\mathrm{p}=0.86\right)$, and we report the results from the factorially invariant restricted model.

We could also parameterize the two sets of cognitive function data as an original or baseline observation at age 79 and an observation of change from age 79 to age 83 based on the difference between the two scores (16). The model of change is effectively identical to the one we used, but the factorial invariance issues are slightly different. In particular, in some situations there may be more power to detect differences in the contributions of the various cognitive tests to change in the overall latent cognitive variable with time using the difference score approach. In this case, however, factorial invariance held and results using this model were effectively identical to those we present, so we do not discuss this approach further. 
Table 1. Mean physical and cognitive test performance at age 79

\begin{tabular}{lrrr}
\hline & Did not attend age 83 & Did attend age 83 & \multicolumn{1}{c}{$\mathrm{t}(\mathrm{sig})$} \\
\hline 6-meter walk time, s & $5.1 \pm 2.4(\mathrm{n}=223)$ & $4.5 \pm 1.5(\mathrm{n}=318)$ & $2.960(\mathrm{p}=0.003)$ \\
Grip strength, kg & $25.3 \pm 8.4(\mathrm{n}=225)$ & $27.4 \pm 9.5(\mathrm{n}=319)$ & $-2.720(\mathrm{p}=0.007)$ \\
FEV $_{1}, 1 / \mathrm{s}$ & $1.8 \pm 0.6(\mathrm{n}=225)$ & $2.0 \pm 0.6(\mathrm{n}=319)$ & $-3.560(\mathrm{p}=0.000)$ \\
MMSE & $27.9 \pm 1.9(\mathrm{n}=229)$ & $28.4 \pm 1.5(\mathrm{n}=319)$ & $-2.892(\mathrm{p}=0.004)$ \\
Raven's Matrices & $29.3 \pm 9.0(\mathrm{n}=225)$ & $32.5 \pm 8.4(\mathrm{n}=318)$ & $-4.303(\mathrm{p}=0.000)$ \\
Verbal Fluency & $38.1 \pm 12.3(\mathrm{n}=228)$ & $41.4 \pm 12.2(\mathrm{n}=318)$ & $-3.090(\mathrm{p}=0.002)$ \\
Logical Memory & $28.8 \pm 12.5(\mathrm{n}=229)$ & $33.7 \pm 12.7(\mathrm{n}=319)$ & $-4.488(\mathrm{p}=0.000)$ \\
\hline
\end{tabular}

Did not attend age 83 = mean age-79 scores for the participants who did not attend the age- 83 follow-up; Did attend age 83 = mean age-79 scores for the participants who returned for the age- 83 follow-up; $t$ (sig) = the $t$ test value (and significance level) for the difference between mean age-79 scores for those who did or did not attend age- 83 follow-up; $\mathrm{FEV}_{1}=$ Forced expiratory volume in $1 \mathrm{~s}$. Figures shown are mean physical/cognitive test scores $\pm \mathrm{SD}$.

Table 2. Mean cognitive ability test performance at ages 79 and 83

\begin{tabular}{llll}
\hline & Age 79 & Age 83 & t (sig) \\
\hline Raven's Matrices $(\mathrm{n}=315)$ & $32.5 \pm 8.4$ & $29.7 \pm 9.2$ & $-8.645(\mathrm{p}=0.000)$ \\
Verbal Fluency $(\mathrm{n}=317)$ & $41.4 \pm 12.2$ & $39.9 \pm 12.7$ & $-3.059(\mathrm{p}=0.002)$ \\
Logical Memory $(\mathrm{n}=318)$ & $33.7 \pm 12.7$ & $33.1 \pm 14.3$ & $-1.049(\mathrm{p}=0.295)$ \\
\hline
\end{tabular}

Figures shown are mean cognitive test scores \pm SD. $t(\mathrm{sig})=$ the $\mathrm{t}$ test value (and significance level) for the difference between age 79 and 83 performance.

\section{Results}

\section{Baseline Sample Characteristics}

Table 1 displays the means for the physical fitness indicators recorded at age 79 separately for those who did or did not attend the subsequent age- 83 follow-up. Compared to participants who were tested at age 79 only, those who returned at age 83 were significantly fitter: their 6 meter walk time was faster, their grip was stronger and their lung function was better. The returning sub-sample also had higher MMSE scores at age 79 (table 1). These data were included for comparative purposes only, and will not be discussed further. Descriptives for Raven's Matrices, Verbal Fluency and Logical Memory performance at age 79 are also presented in table 1. Performance was significantly higher on each of the cognitive ability tests performed at age 79 in those individuals who attended the age- 83 follow-up. This made clear that participants were not missing completely at random, but did not violate the assumption underlying the full informa- tion likelihood approach to our latent growth curve model because continued study participation was in no way contingent on level of performance on these tasks [17].

\section{Cognitive Ability Level}

Table 2 shows the mean cognitive ability test scores at ages 79 and 83 for the returning sub-sample only $(n=315$ for Raven's Matrices, 317 for Verbal Fluency and 318 for Logical Memory). Performance at age 83 was lower on all 3 tests compared with age 79 levels, when comparing the age-79 means of those who attended the age- 83 follow-up. The difference was significant for Raven's Matrices and Verbal Fluency, but not Logical Memory (table 2); participants attending both age-79 and 83 testing sessions thus showed significant decline over 4 years on 2 out of the 3 cognitive ability tests.

The associations among the age-adjusted cognitive ability test measures at ages 79 and 83 are shown in table 3 . The 3 tests given at age 79 showed small to moderate positive correlations, ranging from 0.18 to 0.39 (all 
Table 3. Correlations between cognitive ability tests across the lifespan

\begin{tabular}{|c|c|c|c|c|c|c|c|}
\hline & 1 & 2 & 3 & 4 & 5 & 6 & 7 \\
\hline 1 Raven's Matrices age 79 & - & & & & & & \\
\hline 2 Verbal Fluency age 79 & 0.29 & - & & & & & \\
\hline 3 Logical Memory age 79 & 0.39 & 0.18 & - & & & & \\
\hline 4 Raven's Matrices age 83 & 0.79 & 0.26 & 0.39 & - & & & \\
\hline 5 Verbal Fluency age 83 & 0.32 & 0.76 & 0.24 & 0.36 & - & & \\
\hline 6 Logical Memory age 83 & 0.39 & $0.16^{\mathrm{a}}$ & 0.76 & 0.40 & 0.29 & - & \\
\hline 7 Age-11 IQ & 0.46 & 0.34 & 0.26 & 0.51 & 0.34 & 0.31 & - \\
\hline
\end{tabular}

$\mathrm{n}=537-543$ for the intercorrelations of the age-79 tests, $317-320$ for the intercorrelations of the age- 83 tests, and 313-317 for the correlations between the age-79 and age- 83 tests. The cognitive ability test scores were corrected for age in days at time of testing. Age-11 IQ = the raw age-11 MHT score corrected for age in days at the time of testing and then converted into IQ scores (by definition, age-11 IQ has a mean of 100, and a standard deviation of 15).

All correlations $\mathrm{p}<0.001$, except ${ }^{\mathrm{a}} \mathrm{p}<0.01$.

$\mathrm{p}<0.001)$. The 3 tests also showed moderate positive associations at age $83(0.29<\mathrm{r}<0.40$, all $\mathrm{p}<0.001)$. Performance on any particular test at age 79 was highly correlated with performance on the same test at age 83 (Raven's Matrices $r=0.79$, Verbal Fluency $r=0.76$ and Logical Memory $r=0.76$, all $p<0.001)$. Test performance at age 79 therefore accounted for at least $58-62 \%$ of the variance in later performance on the same test. These percentages may understate the actual common variance because they have not been adjusted for the period-free reliability of the tests. The relatively low correlations among the tests indicated the presence of substantial amounts of test-specific variance.

The association between the age- 79 and age- 83 composite cognitive ability scores was high $(\mathrm{r}=0.84, \mathrm{p}<$ $0.001, \mathrm{n}=313$ ). However, composite scores at the 2 testing occasions differed significantly. For participants completing both assessments, the age-79 mean was 0.19 $(\mathrm{SD}=0.96)$, and age-83 mean was $0.01(\mathrm{SD}=1.02)$; paired sample $t_{312}=5.628, p<0.001$. Thus decline in the general cognitive ability component was observed across the follow-up. Age-11 IQ was positively correlated with both age-79 and age-83 composite cognitive ability scores $(\mathrm{r}=0.49$ and 0.52 respectively, $\mathrm{p}<0.001)$, suggesting that childhood ability accounted for at least $24-27 \%$ of the variance in these composite measures of later life ability.

\section{Linear Regression Analysis of Cognitive Change}

The cognitive change measure obtained by regressing the composite cognitive score from age 83 on the age 79 score correlated $0.15(\mathrm{p}=0.013)$ with age-11 IQ. In a standard linear regression predicting cognitive change from age 79 to 83, our demographic and lifestyle variables sex, years of education, occupational social class, smoking status and alcohol intake were entered simultaneously as independent variables in block 1 . None of these factors was significant at $p<0.05$ in accounting for variance in later life cognitive change (table 4 ), and the overall regression was also not significant $\left(\mathrm{F}_{5,281}=1.040, \mathrm{p}=0.394\right)$. When age-11 IQ was entered in block 2, this accounted for $1.4 \%$ of the variance in later life cognitive change (standardized $\beta=0.138, \mathrm{p}=0.044$; overall $\mathrm{F}_{6,286}=1.558$, $\mathrm{p}=0.159, \mathrm{~F}_{1,280}$ change $\left.=4.094, \mathrm{p}=0.044\right)$. Adding age11 cognition to the regression model thus resulted in a significant increment in the prediction of cognitive change.

\section{Latent Growth Curve Modeling of Cognitive Change}

The parameters that resulted from fitting the latent variable growth curve model are shown in table 5 and diagrammed in figure 1 . The mean slope parameter of -0.03 indicated that cognitive function declined at 0.03 SD per year from age 79 to age 83. Importantly, its variance was very small, only 0.004 (which translates to an SD of 0.06), indicating that most participants experienced about the same rate of cognitive decline. Age-11 IQ did not predict cognitive decline between ages 79 and 83, nor did any of the demographic or lifestyle variables, though male sex, level of education, alcohol consumption, and, especially, age-11 IQ did predict level of cognitive function at age 79 . There was substantial variance 
Table 4. Regression analysis predicting cognitive change from age 79 to age 83

\begin{tabular}{lllll}
\hline & Unstandardized $\beta$ & Standardized $\beta$ & $\mathrm{t}(\mathrm{p})$ & Change statistics \\
\hline Model 1 & & & & $\mathrm{F}_{5,281}=1.04, \mathrm{p}=0.394$ \\
$\quad$ Sex & 0.15 & 0.08 & $1.26(0.208)$ & \\
$\quad$ Education, years & 0.04 & 0.10 & $1.51(0.132)$ & \\
Social class & 0.01 & 0.01 & $0.09(0.926)$ & \\
Smoking, yes/no & 0.20 & 0.05 & $0.86(0.390)$ & \\
Alcohol intake & 0.07 & 0.04 & $0.59(0.557)$ & $\mathrm{F}_{1,280}=4.09, \mathrm{p}=0.044$ \\
Model 2 & & & \\
Sex & 0.13 & 0.07 & $0.12(0.263)$ & \\
Education, years & 0.02 & 0.05 & $0.65(0.517)$ & \\
Social class & 0.06 & 0.05 & $1.02(0.307)$ & \\
Smoking, yes/no & 0.24 & 0.06 & $0.72(0.475)$ & \\
Alcohol intake & 0.09 & 0.04 & $2.02(0.044)$ & \\
Age-11 IQ & 0.01 & 0.14 &
\end{tabular}

Alcohol intake is coded as 1 unit per week or less versus more than 1 unit per week. For the dichotomous variables, the reference categories were male (sex), current smoker (smoking), and 1 unit per week or less (alcohol intake).

Table 5. Latent variable estimates derived from the multiple-indicator linear growth model with time-invariant covariates

\begin{tabular}{|c|c|c|c|c|c|c|c|c|c|c|c|c|}
\hline & 1 & 2 & 3 & 4 & 5 & 6 & 7 & 8 & 9 & 10 & Mean & Variance \\
\hline 1 Age-79 cognition & 1.00 & & & & & & & & & & -0.01 & 0.51 \\
\hline 2 Age-83 cognition & 0.95 & 1.00 & & & & & & & & & -0.15 & 0.59 \\
\hline 3 Model intercept & 1.00 & 0.95 & 1.00 & & & & & & & & -0.01 & 0.51 \\
\hline 4 Model slope & 0.04 & 0.35 & 0.04 & 1.00 & & & & & & & -0.03 & 0.004 \\
\hline 5 Sex & -0.13 & -0.09 & -0.13 & 0.12 & 1.00 & & & & & & 0.58 & 0.24 \\
\hline 6 Social class & -0.40 & -0.35 & -0.40 & 0.07 & 0.10 & 1.00 & & & & & 0.00 & 1.00 \\
\hline 7 Education & 0.48 & 0.44 & 0.48 & -0.03 & -0.12 & -0.48 & 1.00 & & & & 0.00 & 1.00 \\
\hline 8 Age-11 IQ & 0.66 & 0.63 & 0.66 & 0.06 & 0.02 & -0.42 & 0.45 & 1.00 & & & 0.03 & 1.01 \\
\hline 9 Alcohol status & 0.17 & 0.16 & 0.17 & 0.00 & -0.18 & -0.25 & 0.09 & 0.05 & 1.00 & & 0.48 & 0.25 \\
\hline 10 Smoking status & -0.07 & -0.09 & -0.07 & 0.08 & 0.03 & 0.09 & -0.10 & -0.03 & 0.03 & 1.00 & 0.07 & 0.07 \\
\hline
\end{tabular}

For the dichotomous variables, the reference categories were male (sex), current smoker (smoking), and 1 unit per week or less (alcohol intake). The model intercept and age-79 cognition factor were equivalent in the model. The latent means differed slightly from 0 because the model could not completely account for the selectivity in the sample attrition over the 4-year time period. The model slope expressed the change per year. The sex mean of 0.58 reflected the fact that $58 \%$ of the sample was female.

specific to each of the cognitive ability tests, and the variance specific to each test at age 79 was correlated with the variance specific to the same test at age 83 . These correlations were in excess of 0.50 for Logical Memory and Verbal Fluency. The demographic and lifestyle variables (sex, education and alcohol intake), along with age-11 IQ, accounted for $50 \%$ of the variance in level of cognition, but only $3 \%$ of the change in cognition.

\section{Discussion}

The Lothian Birth Cohort 1921 comprises an unusual group of elderly individuals participating in a longitudinal study of cognitive aging, for whom childhood mental ability data are also available. Significant cognitive decline was observed on a composite measure of cognitive ability over 4 years from age 79 to age 83 . Using standard linear regression, mental ability assessed when the cohort 


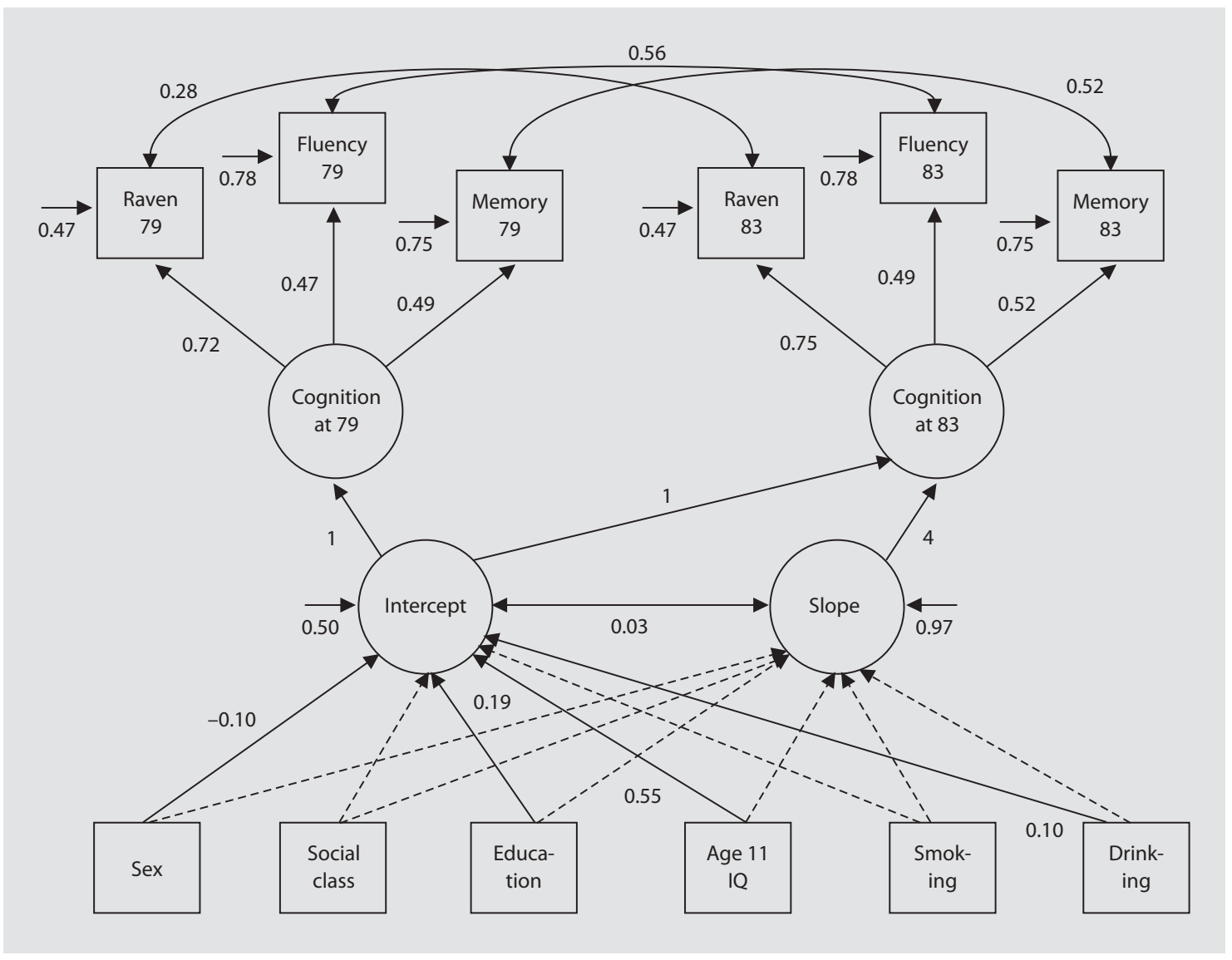

Fig. 1. Latent variable linear growth model with time-invariant covariates. For the covariates, all measured paths are shown, but parameter estimates are given only for those paths that were significant at $\mathrm{p}<0.05$. All parameter estimates are standardized and given to two decimal places. The three parameters shown without decimals were not measured; they were set by the terms of the model to establish the intercept and the time period associated with the slope. Thus, the 4 refers to the 4 -year period between age 79 and age 83 . were aged 11 years old appeared to account for significant variance in later life cognitive change. People who were more cognitively able at age 11 appeared to experience less decline from age 79 to age 83 . In contrast, when a latent variable growth curve model was applied, the association with age-11 IQ was no longer significant. The inclusion of both techniques allows the methodological benefits of the latter to be fully highlighted.

The LBC1921 participants are rare in cognitive aging research due to the presence of a validated measure of prior functioning combined with a substantial follow-up period (currently 72 years). This measure of childhood ability (expressed as an IQ score) was related to performance on the cognitive tests and the composite measures of cognitive ability at ages 79 and 83 , with correlation coefficients ranging from 0.26 to 0.51 . For those partici- pants who were tested at 79 and again at 83 years old, it was possible to investigate the changes in performance on the cognitive measures across this time. At the age- 83 follow-up, the mean levels for Raven's, Verbal Fluency and Logical Memory were lower when compared to the levels 4 years previously, the former 2 significantly so. As with other longitudinal studies of cognitive aging [for example $18,19]$, the members of the LBC1921 experienced detectable cognitive decline in the observation period. This was also found when the 3 cognitive tests were combined in a composite measure. Practice effects would be expected to lead to an underestimate of any effect of aging. In Logical Memory, the actual stimuli may be more memorable, which could explain the lack of significant change observed on this test. However, estimating the absolute amount of change was not the principal aim of the study. 
Rather, our primary purpose was to examine whether ability in early life was associated with the relative amount of change in old age by comparing two commonly available methods.

When we applied classical linear regression, which is often used in such contexts, childhood ability appeared to be significantly related to the change in cognitive ability in old age. Those scoring more highly in childhood appeared to show less decline in old age. This is concordant with several studies that have used similar methodology and that have also had actual measures of early ability and later functioning [20] and change $[4,8]$. This has provided support for the idea that there is 'a strong inverse relationship between early-life... ability and latelife...impairments' [20, p. 345], and suggested that 'ageing effects on intelligence might be less severe on those with high initial ability' [1, p. 361]. Most of these studies, however, have relied on linear regression or similar analysis of variance methodology.

In contrast, when we fit a latent variable growth curve model to the data, we observed no association between change in cognitive function and level of childhood ability. The model parameter estimates indicated that everyone tended to experience a relatively similar, small annual rate of decline of $0.03 \mathrm{SD}$ per year. On an IQ scale, this translates to less than half a point per year, or 4.5 points per decade. The SD of 0.06 in this rate means that some $16 \%$ of the sample actually experienced an increase in cognitive function of at least this magnitude and some $16 \%$ of the sample actually experienced a decrease in cognitive function of at least 3 times this magnitude. Still, the mean rate on which these comparisons are based was so small that it is not surprising that we would not be able to detect any associations with the demographic and lifestyle covariates or with age-11 IQ. We had $99 \%$ power to pick up the change in cognitive function that we did, but age-11 IQ would have had to predict cognitive change with a coefficient of 0.65 for us to pick it up as significant at the $5 \%$ level of significance with $80 \%$ power in this sample. We had an estimated parameter of 0.11 to reach $80 \%$ power at the $5 \%$ level with this effect, we would have required a sample size of more than 10,000 .

It is reasonable to conclude that the results from the latent growth curve model were more accurate than those from the classical regression, despite the fact that the regression results picked up a significant association between age-11 IQ and later life cognitive change and the latent variable growth curve model did not. There are two reasons for this. First, the latent variable growth curve model revealed substantial variance specific to each of the three tests that contributed to the general cognition variable in both approaches. When we created the general cognitive composites using principal component scores for the linear regression, some of this test-specific variance was swept into the general cognitive variable scores because they were linear combinations of the observed variable scores. This test-specific variance was correlated with age-11 IQ because of the underlying association between childhood IQ and later life cognitive function. Some of this test-specific variance may have resulted from practice effects on the tests. Whatever the source, the latent variable growth curve model was able to adjust for the effects because it distinguished test-specific variance from variance common to all three tests. The classical linear regression could not make this distinction.

Second, we created the general cognitive composites for the linear regression using a principal components procedure that estimated the components for the two assessment time points simultaneously. This was necessary because we wanted to be able to use the resulting component scores to measure cognitive change, and a separate analysis would have resulted in separately standardized scores. As described, however, the participants in both assessments had higher cognitive function at age 79 than did those who did not participate at age 83. This higher performance, which was correlated with age-11 IQ, affected the residuals that remained when the age- 83 cognitive function composite was regressed on the age-79 cognitive composite to create the cognitive change scores. The latent variable growth curve analysis was able to make use of full information maximum likelihood estimation that took the missing data at the second assessment directly into consideration, thus circumventing the bias in the estimates created by the selection involved in sample attrition. Creation of the general cognitive composite for the linear regression made it possible to place the analytical focus on general cognitive ability and to remove some of the unreliability of measurement associated with any single specific cognitive measure. The piecemeal nature of the process that included discrete analytical steps, however, was not able to address all the sources of bias. This example emphasizes the importance of making use of analytical techniques that can model the full process under consideration at once. Such techniques are often highly specific to that process.

Despite the lack of association between age-11 IQ and cognitive change between ages 79 and 83 in this sample, it was clear that age-11 IQ was strongly related to age-79 cognitive function. Those with higher IQ scores at age 11 
had better cognitive function at age 79 in this healthy sample. This is important for two reasons. First, for clinical purposes, measures such as the MMSE, on which most younger and many older people generally obtain near-perfect scores, are useful in revealing decline in cognitive function that may impair the ability to live independently. Our latent growth curve model results imply that, because of the small variance in our cognitive change parameter, everyone's cognitive function declined at basically similar rates. This emphasizes the importance, in examining the risks of clinical cognitive impairment, of having some early measure of original level of cognitive function because it implies that that those with higher ability to begin with will, all else being equal, be much slower or less likely to experience this clinical level of cognitive impairment. This is often expressed within the context of the cognitive reserve hypothesis, whereby individuals may experience the same degree of brain pathology, but have quite different overt levels of clinical impairment [21]. The argument is that there is individual variation in the development of clinical impairment of cognitive function and some individuals may have some physical brain structures and/or cognitive strategies that serve as protective reserves against a clinical level of impairment. A higher pre-morbid ability would thus be one potential indicator of greater cognitive reserve.

Second, as in most laboratory studies, we measured cognitive function using artificial tasks that are removed from day-to-day life. In particular, our tasks focused on verbal fluency, abstract reasoning, and logical memory, constructs that place little emphasis on the ability to make use of accumulated knowledge. This was intentional, as performance on tasks that do rely on accumulated knowledge is more closely related to level of education and tends to show relatively little decline with age. But the activities of day-to-day living are not restricted in this way. To the extent that older individuals are aware of decline in some areas of cognitive function, they may make use of accumulated knowledge in ways that successfully compensate for the decline in the activities of day-to-day living for the decline. For example, one could compensate for poorer memory by developing more careful habits of keeping track of one's actions. It is very possible that those with higher original ability are better at arranging this kind of self-compensation [for example, childhood ability (age 11) was shown to be a predictor of later life functional independence (age 77); 22], yet its presence would not be likely to be picked up by our measures of cognitive function.

Mental Ability in Childhood and Cognitive Aging
The LBC1921 participants who did not participate in the second wave of testing were also less physically fit and scored more poorly on the MMSE at age 79. This highlights an area of great complexity in the study of cognitive aging. Many of the physical ailments that are common in old age include cognitive decline in their symptomatology, and many of the participants may have been experiencing both physical and cognitive decline at age 79, thus causing them to withdraw by age 83 . Clearly, decline of this kind may also have contributed to the overall mean decline observed in those who did participate at both ages 79 and 83.

Retention in a longitudinal study such as the LBC1921 appears to be dependent not on early ability but on the declines experienced in later life, though early ability may be associated with initial entry into the study. This selective loss of participants is a limitation of the LBC1921 follow-ups, as it is with any other longitudinal study [23-25]. The cohort under investigation may become progressively biased towards higher cognitive ability and greater physical fitness. While it is impossible to remove this bias entirely, the high response rates achieved with the LBC1921 have reduced it considerably. The LBC1921 did show higher initial levels of age11 IQ than the population from which they were drawn. This is as would be expected from a study of this nature as 'recruitment methods that place high demands on older people, such as volunteering to come to a university for testing, might over-represent the highest performing older adults' [26, p. 88]. Within the LBC1921 Study, however, the presence of early ability data allows this potential confound to be studied explicitly, with respect to the full population, to an extent not possible in other projects.

Though we recorded significant decline, the cognitive tests all display high test-retest associations, highlighting the stability of mental function across time. This stability and the impact of prior ability on later functioning means that discovering determinants of the level of, and change in, later ability is problematical. This is especially true for cross-sectional studies, in which any correlates between putative 'cognitive decline' predictors and cognition are likely to be related to the stable trait of cognition rather than the change over time. The presence of childhood ability allows the investigation of the effect of a range of potential determinants of later functioning whilst simultaneously removing the shared variance associated with this measure. For current purposes, childhood ability and several other covariables were examined in relation to cognitive decline, but there are numerous other poten- 
tial determinants, including health status, activity participation and social support [27].

In a healthy group of older people, we concluded that childhood mental ability was not associated with change in cognitive ability from age 79 to age 83 , though it was associated with level of cognitive ability at age 79. This conclusion was dependent on use of an analytical method (latent growth curve modeling) that could model the full process at once. This does not mean, however, that higher childhood ability may not confer benefits in managing the process of cognitive decline with age in order to preserve the ability to live independently. Addressing this possibility, and the ways in which it might take place, should be a priority of future research, as understanding how individual differences in original level of cognitive ability may be associated with ability to manage daily life tasks in old age could help to identify ways to preserve that ability for a longer time for a larger proportion of the elderly population.

\section{Acknowledgement}

We thank Lawrence Whalley for his comments on an earlier draft of the paper.

\section{References}

1 Deary IJ, MacLennan WJ, Starr JM: Is age kinder to the initially more able? Differential ageing of a verbal ability in the Healthy Old People in Edinburgh Study. Intelligence 1998;26:357-375

-2 Whalley LJ, Starr JM, Athawes R, Hunter D, Pattie A, Deary IJ: Childhood mental ability and dementia. Neurology 2000;55:14551459.

3 Snowdon DA, Kemper SJ, Mortimer JA, Greiner LH, Wekstein DR, Markesbery WR: Linguistic ability in early life and cognitive function and Alzheimer's disease in late life. Findings from the Nun Study. JAMA 1996; 275:528-531.

4 Richards M, Shipley B, Fuhrer R, Wadsworth MEJ: Cognitive ability in childhood and cognitive decline in midlife: longitudinal birth cohort study. BMI 2004;328:552-556.

5 Deary IJ, Whiteman MC, Starr JM, Whalley LJ, Fox HC: The impact of childhood intelligence on later life: following up the Scottish Mental Surveys of 1932 and 1947. J Pers Soc Psychol 2004;86:130-147.

6 Nelson HE, Willison JR: National Adult Reading Test (NART) Test Manual, ed 2. Windsor, NFER-Nelson Publishing Company, 1991.

7 Rabbitt P, Chetwynd A, McInnes L: Do clever brains age more slowly? Further exploration of a nun result. Br J Psychol 2003;94: 63-71.

$>8$ Bourne VJ, Fox HC, Deary IJ, Whalley LJ: Does childhood intelligence predict variation in cognitive change in later life? Pers Individ Dif 2007;42:1551-1559.
9 Scottish Council for Research in Education The Intelligence of Scottish Children: A National Survey of an Age-Group. London, University of London Press, 1933.

10 Raven JC, Court JH, Raven J: Manual for Raven's Progressive Matrices and Vocabulary Scales. London, HK Lewis, 1977.

11 Lezak MD: Neuropsychological Testing. Oxford, Oxford University Press, 1995.

12 Wechsler D: Wechsler Memory Scale - Revised. San Antonio, The Psychological Corporation, 1987.

13 Folstein MF, Folstein SE, McHugh PR: 'Minimental state'. A practical method for grading the cognitive state of patients for the clinician. J Psychiatr Res 1975;12:189-198.

14 General Register Office: Census 1951: Classification of Occupations. London, Her Majesty's Stationary Office, 1956.

15 McGue M, Wette R, Rao DC: Evaluation of path analysis through computer simulation: effect of incorrectly assuming independent distribution of familial correlations. Genet Epidemiol 1984;1:255-269.

16 McArdle JJ, Nesselroade JR: Using multivariate data to structure developmental change; in Cohen SH, Reese HW (eds): Life-Span Developmental Psychology, Methodological Contributions. Hillsdale, Lawrence Erlbaum, 1994, pp 223-267.

17 Little RJ, Rubin DB: Statistical Analysis with Missing Data. New York, Wiley, 1987.

18 Wilson RS, Bienias JL, Evans DA, Bennett DA: Religious Orders Study: overview and change in cognitive and motor speed. Aging Neuropsychol Cogn 2004;11:280-303.

$\checkmark 19$ Christensen H, Mackinnon A, Jorm AF, Korten A, Jacomb P, Hofer SM, Henderson S: The Canberra Longitudinal Study: design, aims, methodology, outcomes and recent empirical investigations. Aging Neuropsychol Cogn 2004;11:169-195.
20 Riley KP, Snowdon DA, Desrosiers MF, Markesbery WR: Early life linguistic ability, late life cognitive function, and neuropathology: findings from the Nun Study. Neurobiol Aging 2005;26:341-347.

21 Richards M, Deary IJ: A life course approach to cognitive reserve: a model for cognitive aging and development? Ann Neurol 2005; 58:617-622

22 Starr JM, Deary IJ, Lemmon H, Whalley LJ: Mental ability age 11 years and health status age 77 years. Age Ageing 2000;29:523-528.

23 Kuller LH: Commentary on 'The NIH Cognitive and Emotional Health Project: report of the Critical Evaluation Study Committee'. Alzheimers Dement 2006;2:33-35.

24 Schaie KW: Developmental Influences on Adult Intelligence: The Seattle Longitudinal Study. Oxford, Oxford University Press, 2005.

25 Hertzog C: Research design in studies of aging and cognition; in Birren JE, Schaie KW, (eds): Handbook of the Psychology of Aging, ed 4. London, Academic Press, 1996, pp 2437.

26 Hedden T, Gabrieli JDE: Insights into the ageing mind: a view from cognitive neuroscience. Nat Rev Neurosci 2004;5:87-96.

$>27$ Hendrie HC, Albert MS, Butters MA, Gao S, Knopman DS, Launer LJ, Yaffe K, Cuthbert BN, Edwards E, Wagster MV: The NIH Cognitive and Emotional Health Project: report of the Critical Evaluation Study Committee. Alzheimers Dement 2006;2:12-32. 